\title{
VERDAD Y LIBRE ALBEDRÍO EN ROBERTO GROSSETESTE
}

\author{
Celina A. Lértora Mendoza \\ Conicet (Buenos Aires)
}

\begin{abstract}
RESUMEN
Son numerosos los filósofos que han tratado sobre las acciones humanas, sobre la relación con la ley natural y la causalidad así como sobre la relación entre conocimiento y el ser. Roberto Grosseteste, en su obra sobre el Libre arbitrio; está especialmente preocupado, a través de más de la mitad de su obra, de la relación entre la libertad y la subsiguiente necesidad de conocimiento divino. Sus reflexiones son un paso más hacia esta cuestión, y pueden ser relacionadas con las respuestas que se han dado posteriormente acerca de este tema siempre actual.
\end{abstract}

Palabras clave: Grosseteste, libre albedrío, necesidad, contingencia, ciencia divina.

\begin{abstract}
Many philosophers have dealt with the subject of freedom of human action and its relation to the natural laws and causality, as well as the relationship between Being and its knowledge. Robert Grosseteste, talking about free will in an homonymus opusculate, starts by asking if free will really exists and is specially worried, through more than half of his work, to the relationship between human freedom and the subsequent need of divine knowledge. His thinking are a step forward towards the question and can be connected to answers which have later been given, about a topic which is always valid.
\end{abstract}

Key words: Grosseteste, free will, necessity, contingency, divine science.

\section{PRESENTACIÓN: PLANTEAMIENTO DE LA CUESTIÓN FILOSÓFICA}

El tema de la libertad de la acción humana y su relación con la necesidad de las leyes naturales es un asunto que ha preocupado a todos los grande filósofos. Aunque se trata de un tema de índole esencialmente antropológica y ética en sí mismo, sus contactos con la metafísica son innegables, de tal modo que a poco se tropieza con un planteo ontológico central: la necesidad causal. Y al mismo tiempo, la relación entre el ser y el conocimiento (cuestión gnoseológica) se introduce también en la medida en que la cuestión de la acción se vincula íntimamente a la de (la posibilidad de) su conocimiento necesario.

La conexión de planos se aprecia apenas se intenta responder a la pregunta: qué es el libre albedrío (o libertad de la acción humana). Y se reafirma en preguntas subsiguientes como: si la acción libre puede ser objeto de conocimiento, de qué tipo de conocimiento, etc. En tercer lugar, parece que sólo suponiendo la libertad (en el sentido de ausencia de determinación necesitante) se puede fundar una moral de premios y castigos (en ésta o en otra vida) y un derecho de obligaciones y prohibiciones de cuyo cumplimiento (o no) se siguen consecuencias relevantes para el individuo (como las sanciones jurídicas).

Desde el punto de vista cotidiano y empírico, podriamos decir que la libertad se manifiesta (a nivel intersubjetivo) sobre todo en la capacidad humana de cumplir o no una orden (cosa que 
no puede hacer un objeto inanimado y muy limitadamente, en cierta forma analógica, el animal superior no humano). Para que una orden pueda ser cumplida se requieren al menos dos condiciones: 1) que el mensaje pueda ser entendido (no lo será si el destinatario no oye, o no entiende el lenguaje, o no tiene suficiente capacidad mental); 2) que esté en su poder total (y no sólo en su_decisión íntima) cumplir o no (así, una persona atada no puede cumplir una orden de moverse, aunque la entienda y quiera cumplirla).

La orden, y la correlativa libertad, están planteando entonces, además de lo ya mencionado, una cuestión de comunicación y de usos del lenguaje. Es obvio que la persona debe entender el tipo de uso del lenguaje que implica una orden, es decir, debe saber diferenciar (aunque sea en su accionar mismo) la diferencia ente el lenguaje descriptivo y el prescriptivo. La lógica muestra las diferencias estructurales (sintácticas) y no sólo semántica, entre ambos usos. La literatura sobre el tema es enorme, y en general se centra en la polémica de si los ámbitos del ser y del deber ser (o ámbito deontológico) son o no reductibles. No vamos a entrar en esta cuestión, pues en general los medievales no se la plantearon así. Pero sí entrevieron la conexión entre o que hoy llamariamos una lógica deóntica (que es necesariamente modal) y la lógica modal que conocían, la de las modalidades aléticas aristotélico-estoica. De allí que la cuestión se plantee en términos de necesidad/posibilidad, lo que nos ancla definitivamente en terreno ontológico.

\section{EL TEMA SEGÚN ROBERTO GROSSETESTE}

Roberto Grosseteste es uno de los autores que decididamente enfoca el tema del libre albedrío en el sentido indicado, en su obra De libero arbitrio, donde la cuestión comienza por la usual pregunta «si existe» ${ }^{1}$, reconociendo que hay fuertes razones tanto en uno como en otro sentido. La disputa enfoca como punto central, el problema de la contingencia en el universo, en la medida en que ella se incluya en la definición de libertad.

En términos generales, se dice que en un proceso no hay libertad cuando los estadios anteriores en el tiempo determinan en forma total y absoluta lo que va ocurriendo en los estadios posteriores. Es necesario que el proceso posterior esté determinado totalmente para declarar la falta de libertad de un resultado; un proceso puede estar parcialmente determinado y ser a la vez libre en la medida en que no está determinado. Así definido un proceso libre, está claro que el acto libre es esencialmente contingente. Por lo tanto, si existen actos libres existen actos contingentes, lo cual supone la refutación del determinismo universal.

De allí que los partidarios del libre arbitrio confrontan en primer lugar con los deterministas, que sustentan el determinismo universal. A su vez, la postura determinista tiene diversos grados y matices. En su forma más extrema opone la determinación al azar. Aunque no es la única manera de entender la oposición, es la que la exhibe con más fuerza. Sin embargo, el hecho de que una conducta o acontecer no sea totalmente determinada (determinismo en sentido estricto) no significa que sea absolutamente azarosa. En este sentido la oposición se vincula con la oposición causa-azar (falta de causa).

Verdad y necesidad: crítica al determinismo. Ahora bien, desde el punto de vista de Grosseteste - aunque no el único medieval que lo sustenta - hay una estrecha relación entre el determinismo que supone ontológicamente la necesidad causal y su expresión lógica correspondiente a la verdad apodíctica de las proposiciones universales. Si asumimos, como lo exige la teología, que Dios conoce absolutamente todo y que su conocimiento es absolutamente necesario (en el sentido de que no puede no ser), entonces todo sucede necesariamente, ya que

1 Se cita por la ed. L. Baur, Die philosophischen Werke des Rohbert Grosseteste, Bishofs von Lincoln, Münster, 1912, p.150. 
incluso los que son para nosotros futuros contingentes o condicionados, son necesarios en cuanto conocidos por la mente divina.

Un párrafo atribuido al Lincolniense, en otra recensión del De libero arbitrio, expone la tesis en su versión maximalista: «Como Dios conoce todo, incluso los futuros contingentes, su ciencia sobre ellos es inmutable e infalible; por lo cual, cuando conoce algo, es necesario que [eso] suceda, para que su conocimiento no sea falible o mutable, por tanto todo sucede necesariamente ya que [Dios] no puede no conocer lo que conoce. Luego nada procede del libre albedrío de las creaturas, porque si así fuese, no sería necesario» ${ }^{2}$.

La verdad de los futuros contingentes. La cuestión se plantea entonces en relación a la verdad de los futuros condicionados, el conocimiento divino de los contingentes (incluyendo sobre todo la predestinación) la profecía, la adivinación y la gracia. Para fundamentar la verdad de la afirmación de la existencia del libre albedrío, más de la mitad del tratado se dedica a establecer la compatibilidad entre la necesidad eterna de Dios y de su conocimiento, y la contingencia requerida por la acción libre.

Este tema se plantea análogamente desde perspectivas no religiosas, como compatibilidad (o no) entre la necesidad causal universal y la contingencia de un hecho concreto. Para quienes rechacen la posición extrema (y científicamente poco atractiva) de fundamentar la existencia de la libertad como azar, negando todo tipo de determinación ontológica, se trataría de hallar un compromiso que permita usar la categoría de causalidad sin conectarla ineludiblemente al determinismo absoluto.

Una manera de introducir la causalidad en el discurso, sin compromisos ontológicos, es considerarla una condición (en sentido lógico) necesaria o suficiente. Tendremos así los siguientes casos

1. condición suficiente:

si $\mathrm{p}$ entonces q (no descarta otras causas de q: por ejemplo si llueve me mojo, pero puedo mojarme por otras causas)

2. condición necesaria

si se dio $q$, entonces se dio $\mathrm{p}$, pero no descarta que no ocurra la consecuencia (puede no ocurrir, y en ese caso nada se infiere sobre $p$ ).

Entonces, sin plantear la cuestión ontológica en términos de causalidad, sino sólo de ocurrencia, podemos establecer modalidades aléticas vinculadas a las ontológicas, pero no comprometidas con la relación de causalidad entre conocimiento (divino) y ocurrencia (contingente). Grosseteste asume la cuestión en términos análogos, haciéndose eco de una problemática que ya habían abordado Boecio y San Anselmo.

Veamos la crítica al determinismo, que es el argumento fundamental de los negadores del libre albedrío ${ }^{3}$. Para el determinista, todo lo que ocurre, ocurre necesariamente. Si hubiera una excepción el determinismo sería falso ${ }^{4}$. Una crítica al determinismo proviene del análisis de

2 Recensio altera (Ex) de c. 1, 31-35 (Baur, p. 151).

3 Históricamente los estoicos, con Crisipo, sostuvieron el determinismo universal, y para el caso de la acción humana incorporaron a la cadena causal el carácter y las inclinaciones del agente. En la modernidad, Hobbes y Spinoza son deterministas en el sentido estoico, y por tanto la libertad se reduce a ausencia de impedimentos externos para hacer lo que se quiere (determinadamente). En contra, San Agustín defiende la libertad del hombre, incluso frente a Dios. Para Descartes el libre albedrío es simplemente algo evidente, no lo tematiza ni lo demuestra. Kant retoma el tema pero afirmando que hay libertad sólo para cumplir con la ley moral.

4 En esta formulación las teorías se excluyen mutuamente. Sin embargo, en otros contextos filósofos, entre ambas teorías opuestas se colocan algunas intermedias:

La teoria de la sobredeterminación: el agente libre, al elegir, no niega la causalidad universal sino que agrega una determinación para el efecto respectivo. Pero en realidad esta tesis contradice la causalidad universal y por tanto en el fondo es indeterminista. Si el esquema determinista es tlp-t2q ( $p$ es causa, $q$ es efecto en los tiempos 1 y 2 y es el condicional material, el esquema de la sobredeterminación sería $\mathrm{t} 1 \mathrm{p} . \mathrm{a}-\mathrm{t} 2 \mathrm{q}$, donde a es el acto del agente, pero si a no pertenece a la cadena causal se contraria el determinismo universal, y si pertenece, entonces el acto del 
las proposiciones de futuro contingente, planteo que analizó Aristóteles con el ejemplo de la batalla naval que podía o no tener lugar al día siguiente. Dado que ambas proposiciones son contradictorias, por fuerza una tenía que ser verdadera y de allí se podría inferir que los hechos futuros no son contingentes porque podemos hablar con verdad acerca de ellos. Los hechos futuros están predeterminados como necesarios o imposibles y la apariencia de su contingencia es sólo producto de nuestra ignorancia.

En relación al conocimiento, el silogismo determinista toma esta forma

«Todo lo conocido por Dios o es, o fue o será

A es conocido por Dios - siendo A un futuro contingente-

Luego A es, o fue o será -Pero no es ni fue

Luego será» ${ }^{5}$.

Pero para que la tesis determinista sea verdadera, la conclusión debe ser necesaria. Esto se debe a que, para quienes - como Grosseteste, siguiendo a Aristóteles- aceptan la noción de verdad como adaequatio ${ }^{6}$, la expresión proposicional debe exhibir la modalidad alética equivalente estatuto ontológico de lo expresado. En nuestro caso, ambas premisas son necesarias, de lo cual en principio, los lógicos deducen que la conclusión también lo es, conforme al principio «ex necessariis non sequitur nisi necessarium» que Grosseteste refiere allí mismo. Por lo tanto, para negar la necesidad de la conclusión deben darse algunos de estos supuestos: a) que alguna de las premisas sea falsa; b) que el condicional no es necesario; c) que no valga el principio, y que de lo necesario se siga también lo contingente.

a) Rechaza la primera porque implicaría sostener que Dios desconoce algo, y entonces no sería omnisciente. Sin embargo, esta solución tenía venerable tradición: Aristóteles había afirmado que la ciencia es de los universales; Dios tiene pues, ciencia de los universales, no de los singulares en tanto tales. Pero un autor cristiano no puede apoyar esta afirmación ${ }^{7}$. Hay otra versión de esta tesis: no se niega que Dios conozca A (es decir no se toma «Dios conoce A» como falsa) pero se niega que conozca A necesariamente. Entonces si no es necesario que Dios conozca A (porque podría no conocer lo que conoce) el antecedente no es necesario (porque una de las premisas no lo es) y por tanto tampoco puede serlo el consecuente. Grosseteste dedica varios párrafos a establecer la falsedad y la imposibilidad de la proposición «no es necesario que Dios conozca A» porque implica que Dios no conocería lo que conoce.

Se propone pues, impugnar dos soluciones. Primera: la proposición «Dios conoce A», no es necesaria, si A es un singular contingente, porque si A no fuera Dios puede no conocerlo. Segunda: la proposición no es necesaria porque puede no conocer A antes que A sea. Por tanto, así como $\mathrm{A}$ es o no es, así conoce o no conoce $\mathrm{A}^{8}$. La primera solución es inaceptable: decir que la proposición no es necesaria es impio porque implica que Dios mutaría en cuanto a su ciencia, y para probarlo puede apoyarse en numerosas citas de San Agustín y Dionisio, y además se puede probar con argumentos.

agente no es libre. Por lo tanto, en realidad la alternativa estricta se mantiene, ya que las «terceras posiciones» se reducen a uno u otro de los términos opositivos.

5 Ibid. p. 152.

6 En realidad Grosseteste habla, en el conjunto de su obra, de dos aspectos de la verdad: la verdad ontológica (id quod est) tal como lo trata en su obra De veritate y la verdad como relación justa y correcta, como adaequatio. Aunque en este último sentido se aproxima a Aristóteles (precisamente la expone en su comentario a los Posteriores Analíticos), su visión en general depende en gran medida de Agustín y Anselmo (cf. R. J. Oalma, «Robert Grosseteste's Understanding of truth», Irish Theol. Quart. 52, n. 4, 1974: 300-306 y J. Mc Evoy, «La connaissance intellectuelle selon Robert Grosseteste, Rev. phil. Louvain 75, n. 25, 1977: 5-48.

$7 \quad$ De libero arbitrio, p. 152.

8 Ibid. recensio altera, p. 161. 
La argumentación que presenta la segunda versión es interesante porque implica un esfuerzo por establecer una relación coherente entre la realidad y la ciencia acerca de ella tanto en el plano humano como en el divino. Propone el siguiente esquema':

$\frac{\text { «sentarme» }}{\text { me siento }} \cdot \frac{\text { «haberme sentado» }}{\text { me senté }}=\frac{\text { ciencia divina }}{\text { me siento }} \cdot \frac{\text { ciencia divina }}{\text { me senté }}$

Es decir: así como en el orden de nuestro conocimiento la enunciación verdadera se relaciona con el orden real pasado o presente, así la ciencia divina se relaciona — sin cambiar ni mutar ella misma - de manera distinta a nuestra realidad según que sea presente o pasada. Dios conoce los futuros como presentes, y todo (pasado, presente, futuro) lo conoce de la misma manera y con necesidad.

Aquí se introduce una distinción, entre necesidad antecedente y consecuente, que servirá para explicar que el conocimiento necesario divino del futuro no cambia el carácter contingente del mismo. Se basa para ello en la posición de San Anselmo en Cur Deus homo y De concordantia praescientia Dei cum libero arbitrio. También vincula su solución a la distinción boeciana entre necesidad simple y necesidad condicionada. Según él, Boecio, para estos casos, habla de necesidad absoluta y condicional (De consol. Phil. V, 6) aunque por «condicional» no entiende la necesidad consiguiente, sino la necesidad de consecución entre antecedente y consecuente (ejemplo: si el hombre corre, mueve los pies). Aclara Grosseteste: «Él llama 'necesidad de condición' a lo que llamamos 'necesidad consiguiente', diciendo: 'el futuro, en cuanto referido a la noción divina, es necesario, en cuanto a su propia naturaleza parece ser libre'. Y poco después afirma que Dios contempla como presentes las cosas que provendrán del libre

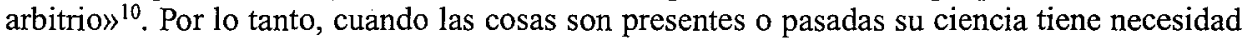
consecuente; y lo mismo es para las futuras.

Sin embargo, lo que intuitivamente se ve claro con relación al pasado o al presente, no parece ser tan claro con respecto al futuro, que es el punto que nos interesa. Volveremos sobre esto.

La segunda solución considera que Dios puede no conocer A mientras A no existe, ya que la ciencia versa sobre lo existente ${ }^{11}$. Pero si la ciencia divina dependiera de la existencia de las cosas temporales, sería mutable, lo que es falso e impío, porque todo lo que Dios conoce, lo conoce eternamente ${ }^{12}$. El argumento es el siguiente: «Dios conoce A. Luego puede conocer A o no puede ${ }^{13}$. Si no puede no conocer A, entonces necesariamente conoce A. Si puede no conocer A, entonces puede pasar de conocedor a no conocedor de A. Luego puede cambiar y alterarse, lo que es imposible» ${ }^{14}$.

Puede aducirse un confirmación gnoseológica del anterior argumento, por reducción al imposible: el ente que no puede dejar de ser no puede no ser, sino que es necesario. Pero existe la verdad de estas proposiciones: «Dios conoce A», «el Anticristo es futuro», «Es verdad que el Anticristo será», «El Anticristo existirá». Ninguna de estas verdades puede dejar de ser. $Y$ si pudieran dejar de ser, entonces estas verdades no son continua, no fueron desde la eternidad,

9 Ibid. p. 163.

$10 \quad$ Ibid. p. 159.

11 Ibid. p. 161.

12 Ibid. p. 163.

13 Aunque parece extraño, el paso es lógicamente correcto: una proposición verdadera p mantiene su valor de verdad puesta en disyunción inclusiva con cualquier proposición, sea verdadera o falsa. Luego si «Dios puede conocer A» es verdadera, la proposición «Dios puede conocer $\mathrm{A}$ o no puede conocer $\mathrm{A}$ » también lo es.

14 Ibid. p. 164. 
es decir, no son eternas. Pero se ha supuesto que lo son, luego a la vez son y no son eternas, lo que es imposible ${ }^{15}$.

El mismo argumento puede expresarse, en sentido inverso, partiendo de la suposición de la falsedad: si esta verdad falla (la de cualquiera de las proposiciones de futuro contingente), en eso mismo comienza la falsedad. $Y$ la falsedad significa que eternamente fue falsa. Entonces su falsedad no tuvo comienzo. Luego, si comenzó, no comenzó. Y por tanto el comenzar mismo es imposible. $Y$ su opuesto, al verdad, es necesaria. Luego esta proposición es necesaria: «El Anticristo será...» del mismo modo que cuando digo «El Anticristo es futuro», su futurición no puede dejar de ser, y del mismo modo que el Anticristo es futuro, es necesario y el Anticristo necesariamente es futuro ${ }^{16}$.

b) La posibilidad b no es tomada especialmente en cuenta por Grosseteste porque en una lógica fuerte o bivalente no se admiten conexiones disminuidas. Es decir, una implicación lo es o no lo es. Si es una implicación, juegan inmediatamente las modalidades aléticas.

c) La posibilidad que explorará el Lincolniense es la tercera. Y el caso crítico para establecer alguna diferente interpretación del principio es justamente el de los futuros contingentes, tema que el Lincolniense aborda en su opúsculo De veritate propositionis. Si bien el tema forma parte de una preocupación más general y amplia, el tema de la verdad (al cual dedica el De veritate) y en ambos las fuentes decisivas son Agustín y Anselmo ${ }^{17}$, el objetivo y el tema específico en ambas son distintos, pues mientras que en De veritate se trata de definir el concepto de verdad en sentido ontológico, en De veritate propositionis atiende a un caso especial, que es el de los futuros contingentes, para establecer si y en qué sentido puede aplicarse a las proposiciones temporales en materia contingente los resultados de su análisis ontológico. Importa señalar que ambas obras omiten referencias significativas a Aristóteles aún cuando el comentario del Linolniense a los Posteriores Analiticos fue escrito contemporáneamente, o incluso con anterioridad. Está claro que en estos dos tratados expone Grosseteste no sólo su propia teoría de la verdad, sino que ésta resulta una solución universal (en visión agustiniana) frente a las lagunas de la teoría aristotélica reducida a la verdad científica empírica. Por tanto no es extraño que en relación a nuestro problema, Grosseteste no se sienta atado a la teoría veritativa aristotélica.

La cuestión de los futuros contingentes es abordada en De veritate propositionis a partir de la relación entre el hecho y el enunciado que lo exhibe. La necesidad del enunciado y de la conexión lógica entre premisas y conclusión deriva del estatuto ontológico de los hechos. El siguiente texto, paralelo al mencionado antes, es claro en cuanto a esa relación: «Una cosa que en parte es o fue y en parte es futura, no necesariamente existe totalmente antes de que su complemento exista o haya existido. Pero como lo completado es algo, entonces también con respecto a lo otro es necesario que sea o haya sido en sentido absoluto. Entre el comienzo y el complemento es necesario que éste en algún sentido sea o haya sido. Y así, las cosas contingentes, que suceden en el tiempo, es posible que no se completen» ${ }^{18}$. El ejemplo propuesto ilustra, con el caso del movimiento, la posible secuencia de un proceso, es decir de un hecho que se dilata en el tiempo. Tomemos que $\mathrm{C}$ es el movimiento total entre dos extremos: A y B. Entre el inicio y el fin de $\mathrm{C}$ necesariamente hay movimiento. Si tomamos un punto intermedio ente A y B, C ya ha sido, pero es posible que $C$, en cuanto a su parte futura, no sea, por ejemplo, porque se interrumpa el movimiento antes de llegar a $\mathrm{B}$, ya que este movimiento es contingente. Hasta aquí la descripción de los hechos. Veamos ahora cómo los expresamos mediante una proposición que sea determinadamente verdadera.

15 Ibid. p. 164.

16 Ibid. p. 165.

17 Cf. J. Mc Evoy, The philosophy of Robert Grosseteste, Oxford, Clarendon Press, 1986, p. 230.

18 Ed. Baur, Werke, p. 143. 
Entre el inicio y el fin de $\mathrm{C}$, el movimiento siempre se predica verdaderamente, porque $\mathrm{C}$ está sucediendo. Pero éste no es un ser completo, determinado y definido, porque no ha llegado todavía a $\mathrm{B}$, sino que es incompleto, no-terminado e indefinido. Por lo cual, en la mitad del movimiento de $\mathrm{C}$, cuando se dice verdaderamente que $\mathrm{C}$ existe, se puede decir consiguientemente: «es necesario que $\mathrm{C}$ haya sido», porque, cuando $\mathrm{C}$ es presente, una parte suya es pasado y la otra es futura, y $\mathrm{C}$ se dice verdaderamente necesario de su parte pasada, porque ahora existe. $Y$ en cuanto es pretérito, es perfecto. Pero, por otra parte, $C$ tomado como un todo ( $\mathrm{y}$ no en cuanto a una parte) antes de su fin ni es pretérito, ni es perfecto. $Y$ tampoco se puede decir verdaderamente que $\mathrm{C}$ como un todo haya sido, porque ahora esté sucediendo, $\mathrm{y}$ así será mientras que en el futuro no haya sido $^{19}$.

Diríamos entonces que tomando $C$ en un punto anterior a su fin, la proposición «C existe» tiene dos modalidades: aplicada al pasado es necesaria, aplicada al futuro es posible. Es decir, que una es la verdad en relación al pasado y otra al futuro, cuando se trata de entes contingentes, admitiendo que verdad es adecuación de la proposición a la realidad, ya que pasado y futuro no son lo mismo ${ }^{20}$. Y así, la verdad acerca del futuro en cuanto a lo que ya es, es necesaria, y en cuanto a lo que no es, es contingente. De modo que Grosseteste define lo posible como equivalente a lo contingente, en el sentido de que no es necesario ni imposible.

Sin embargo, pareciera que el Lincolniens no comprendió la ambigüedad de la definición Aristóteles de «posible» ${ }^{21}$ y de allí esta solución que deja puntos oscuros. Pero en lo que hace a la cuestión del principio ex necessariis, Grosseteste la ha enfocado de manera significativa, tomando el toro por las astas y planteado la alternativa al mismo. Al referirse a la objeción del conocimiento profético como determinado (un argumento contra el libre albedrío) dice que de una misma manera se resuelven las objeciones relativas a la profecía y a la adivinación. Pues parecen oponerse porque en estos dos casos hay contingencia hacia ambas posibilidades, pero a la vez hay necesidad, lo que no permite afirmar la contingencia del consecuente.

En efecto, la cosa profetizada de sí es posible hacia las dos partes (suceder o no suceder), y es la presciencia profética la que no admite que una de ellas sea. Por lo tanto es necesario que suceda esta cosa conocida, de cuyo conocimiento se sigue el ser de la cosa, que sin embargo es contingente. La cual contingencia, como se ha dicho, no parece permitir la necesidad del antecedente, sino más bien destruirla ${ }^{22}$.

Según Grosseteste, la solución de esta dificultad está en determinar de qué modo la necesidad del antecedente destruye y de qué modo permite la contingencia del consecuente. Para esto formula una regla alternativa al principio anteriormente enunciado (de lo necesario sólo se sigue lo necesario). Así como de lo necesario parece seguirse lo contingente, así, de lo contingente parece seguirse lo imposible, porque del opuesto del contingente consiguiente necesariamente se sigue el opuesto de aquel necesario ${ }^{23}$. Veamos con más detenimiento esta formulación.

Primera afirmación: de lo necesario se sigue lo contingente, El mismo Libncolniense da poco más adelante un ejemplo ${ }^{24}$ : es contingente que esta hierba florezca mañana, pero el co-

\section{Ibid. p. 143.}

20 Es la soluciones que propone en De veritate propositionis: «Pues la verdad de la proposición o de la opinión es la adecuación de la proposición u opinión a la cosa. Y esta adecuación no es sino que la cosa es de tal modo como la proposición u opinión dice, y esto es que la cosa sea en el futuro, como la proposición u opinión afirman que será en el futuro. Luego, hay una verdad de la proposición u opinión presente con respecto al futuro de la existencia de la cosa en el futuro con la existencia de la cosa misma en el futuro» (ibid. p. 144)

21 Cf. J. Dawson, «Necessity and Contingency in the 'Libero Arbitrio' of Grosseteste», Filosofia della natura nel Medioevo, Atti del $3^{\circ}$ Congresso Internazionale. de Filosofia Medievale, Milano, 1964, pp. 357-362.

22 Ibid. p. 160.

23 Ibid. p. 160 (Recensio altera)

24 Ibid, p. 167 (recenso altera) 
nocimiento de este florecimiento en la mente divina es necesario. Luego de esta razón eterna y necesaria se sigue un hecho contingente. Destaquemos, por supuesto, que este «sequitur» en sentido ontológico sólo es válido aceptando la relación causal del creador respecto a la creatura (y no del cognoscente respecto a lo conocido). Para que la conexión entre conocimiento y realidad valga en sentido general para todo acto cognoscitivo, sería necesario que el cognoscente tuviera la posibilidad de un conocimiento cierto, determinado y absoluto (lo cual, por hipótesis no se da en el hombre, pero sí en Dios, que es el caso-límite referido al conocimiento del futuro, ya que la ignorancia humana al respecto es obvia).

segunda afirmación: de lo contingente se sigue lo imposible. Tomemos un ejemplo:

Si es A, luego es B - siendo A contingente, B también lo es;

Luego si es no-B, se sigue no-A: el opuesto contingente de $B$ determina que necesariamente es no-A.

Pero si no-A es necesario B es imposible.

Grosseteste admite que esto parece contrario a las reglas de la dialéctica (de la lógica aristotélica) según las cuales de lo necesario no se sigue lo contingente, sino sólo lo necesario y que de lo contingente no se sigue lo imposible y que cualquier necesario es compatible con todo contingente ${ }^{25}$. Entonces se trata de saber exactamente en qué caso se aplica este principio lógico, y opina que quizá esto sea válido para el necesario antecedente y absoluto, pero no para el necesario consiguiente y condicionado. Pero no aseguro que sea así, puede no serlo aunque no parezca absurdo. Grosseteste advierte que no tiene una solución directa para el problema, y por tanto procura una solución indirecta, refutando soluciones falsas, como son todas aquellas que terminan implicando la contradicción de que Dios conoce lo que no conoce, si la ciencia divina se hace depender de la existencia temporal de lo conocido.

Ahora bien, si nos atenemos a la necesidad inmutable del antecedente, no podemos admitir la excepción propuesta al principio ex necessariis. En efecto la ciencia sobre una cosa pasada no puede no haber sido y en esto no vale que alguien alegue que de lo necesario se sigue lo contingente, como se indicó antes. No puede ser, porque si el antecedente no puede dejar de ser verdadero, tampoco el consecuente puede.

Llegado a este punto, Grosseteste no oculta la perplejidad: «En lo ya dicho parece haber obstáculos por doquier, por eso hay que salir de ellos.'Pues parece que todo lo verdadero futuro es necesario y al contrario, que los futuros corruptibles son contingentes» ${ }^{26}$.

Una vía de esclarecimiento y eventualmente de solución es distinguir los sentidos en que se usa lógicamente la modalidad de necesidad. Según uno de esos usos parece claro que de lo necesario se sigue lo contingente y contra esto no hay argumentos silogísticos (es decir, prueba o demostración directa en contrario). En sentido absoluto, no se puede evadir la imposibilidad de que lo mismo y según lo mismo se diga a la vez contingente y necesario, porque si es necesario no puede no ser, y si es contingente puede no ser. Y esta contradicción simultánea no puede ser. Pero no hay contradicción si se distinguen, y se toma «necesario» en el sentido preciso de que lo que no es no puede relacionarse ni al no-ser ni al no-haber-sido, ni al nohaber-de-ser, así como es necesario que dos más tres sean cinco, porque con relación a esto no es posible ni en el pasado, ni en el futuro, ni desde siempre, ni nunca, que no sea verdad. Por lo cual esto es necesario en sentido absoluto, y de ningún modo su no-ser puede ser o llegar a $\operatorname{ser}^{27}$.

25 Esta regla es válida en la lógica modal contemporánea: una proposición necesaria en disyunción inclusi-. va con una contingente da por resultado una fórmula molecular necesaria.

26 Ibid, p. 168 (recensio altera).

27 Ibid. pp. 168-169 (recensio altera). 
En cambio, es necesario de otro modo, lo que cuando es no tiene poder para tener él mismo no-ser después del ser que tiene. Así las proposiciones verdaderas de pretérito claramente son necesarias y este necesario se sigue del necesario anteriormente mencionado.

Por tanto lo necesario absoluto se divide en este necesario y en necesario que-no puede no ser después de ser en relación al eterno poder nunca ser, o haber-sido o haber-de-ser. Tal es la vęrdad de los dichos de futuro, porque la verdad de ellos, cuando es, no puede tener no-ser después del ser, como se ha demostrado. Sin embargo, en relación a los que eternamente fueran falsos, su posibilidad de falsedad no tuvo inicio. Es decir, que como estas proposiciones tienen determinada verdad y falsedad, no tiene posibilidad a su opuesto, en sentido temporal. Y esto se aplica al caso del conocimiento divino. Porque cuando Dios conoce algo, no es que pueda conocer en el sentido de que después de haber conocido algo, no lo conozca, pero sí es eterna posibilidad de que eternamente no haya conocido lo que conoce ${ }^{28}$. En definitiva, y aunque con ciertas vacilaciones lingüística y conceptuales, Grosseteste quiere decir que la posibilidad ontológica (en el sentido de contingencia) no se opone a la necesidad lógica (conexión necesaria de antecedente y consecuente) ni tampoco la temporalidad de los hechos (la realidad que exige la adaequatio) guarda paralelo lógico con la posibilidad de negación de la proposición necesaria.

De este modo se puede concluir que el conocimiento determinado y necesario de los futuros contingentes no destruye la contingencia de ellos mismos convirtiéndolos en necesarios y por lo tanto la ciencia divina es compatible con el indeterminismo del futuro, que es el requisito ontológico para que pueda haber libre albedrío. No se ha planteado este tema, en cambio, en términos de predestinación y su relación con la gracia y el merecimiento, como siglos después lo hará Lutero y otros reformadores. Es claro que la preocupación del Lincolniense aquí no es legitimar un libero arbitrio frente a un servo arbitrio, sino mostrar que no hay razones ni argumentos lógicos que puedan demostrar en forma apodíctica el determinismo.

\section{VIGENCIA DE LA DISCUSIÓN.}

El tema del libre albedrío, y la oposición al determinismo universal no es un asunto histórico; al contrario, posiciones seculares se renuevan con nuevos argumentos. Así, la tesis fuerte del determinismo es contestada actualmente de diversas maneras.

Desde el punto de vista de la lógica contemporánea, Von Wrigth conserva las proposiciones de futuro, pero introduce una variante en el razonamiento al señalar que en ellas el modalizador temporal de futuro no se distribuye. Por otra parte, las proposiciones de futuro serían dudosamente proposiciones ya que en ellas aún no existe el referente de la proposición.

Desde la macrofísica, con implicancias cosmológicas y filosóficas, el argumento de Prigogine afirma que el árbol topológico correspondiente al pasado tiene forma lineal, pero con respecto al futuro podría estar ramificado, y de esas posibilidades sólo una puede realizarse. Esto significa que no hay determinismo estricto u ontológico en relación al futuro.

Ahora bien, si no se presupone el determinismo universal, para cualquier proposición de futuro hay tres posibilidades: 1 . que sea verdadera en todos los mundos posibles (tautología); 2. que sea falsa en todos (contradicción); 3. que sea verdadera en unos y falsa en otros, pero en este caso no puede atribuírsele ningún valor hasta su efectiva ocurrencia (el «indemostrable» de los lógicos del s. XIV). Observemos que Grosseteste ha intuido este estatuto variado de las proposiciones de futuro contingente al menos quoad nos, aunque lo ha expresado en términos de necesidad antecedente y consecuente. Se trata, en definitiva, de establecer una es- 
trategia que permita superar la sobredeterminación del tipo de conocimiento (necesario en sentido absoluto, para Dios) en relación a los sucesos individuales reales.

Hay en cambio un punto en que la filosofia contemporánea no religiosa se distancia de los medievales, y es su prescindencia sistemática del tema del conocimiento absoluto divino con lo cual se evita cierta confusión entre «libertad» y «omnipotencia», que ocurre cuando se prescinde del hecho de que la libertad de elección se ejerce dentro de un repertorio de posibilidades de carácter finito.

En este sentido podemos dar una lectura no teológica de la «predestinación». Tomemos el caso del pecado original: si Dios hubiese querido que un estado de cosas determinada por Él fuese inamovible, entonces lo habría sustraído al libre albedrío de los agentes, por ejemplo el acto de Adán de comer o no comer la fruta. Por lo tanto Dios, ateniéndonos al relato del Génesis, deberíamos decir que no quería en forma incondicional la no-ingesta del fruto. Él quería que la no-ingesta fuese un acto del hombre. Por lo tanto, del relato pueden extraerse dos consecuencias importantes para la teoría de la acción: 1. hay acciones no mencionadas por Dios como regladas y su repertorio es en principio ilimitado; 2 . las acciones no mencionadas están permitidas. Por lo tanto, en el sexto día, cuando crea al hombre, Dios se desposee del poder que transfiere al hombre y así algunos estados futuros del mundo dejaron de ser competencia exclusiva de la voluntad Dios para pasar a ser competencia del hombre o de otros seres creados. En este caso, la filosofía, o la teología, deberán explicar qué tipo conocimiento tiene Dios de esas posibles futuras acciones, pero cualquiera sea la respuesta a este interrogante, no compromete la compatibilidad entre una causalidad universal necesaria general y sectores más o menos amplios (piénsese en la teoría de Prigogine, por ejemplo) sustraídos a ella. Grosseteste, en cambio, como otros medievales, consideraba necesario mantener la conexión entre los dos ámbitos, de allí el esfuerzo por aproximar las soluciones lógicas a las onto-teológicas

Digamos finalmente, que el tema de la omnisciencia divina, si bien es un tema teológico, implica una cuestión filosófica decisiva: si el libre arbitrio de un agente A es compatible con un conocimiento de sus acciones futuras por parte de otro agente o sujeto. Esta cuestión decisiva fue claramente vista, expuesta, desarrollada y contestada por Grosseteste, con los recursos teóricos que brevemente he mencionado.

Pero su solución, que le interesaba sobre todo en cuanto teólogo, tiene para nosotros además un interés epistemológico. El recurso al conocimiento de Dios puede ser considerado como el caso teórico-límite de la relación entre conocimiento y agencia libre. En otros niveles, otros sujetos de conocimiento estarán en posiciones análogas (no por supuesto, iguales). Es el caso de los estudios e investigaciones sobre el comportamiento humano. En las actuales ciencias sociales se admite que el científico tiene un cierto conocimiento de las acciones futuras de agentes libres, pero como tendencia o en general, todo lo cual es compatible con el ejercicio individual del libre arbitrio. De esta situación que puede aceptarse como conocimiento imperfecto del accionar futuro libre, no se sigue ninguna consecuencia ontológica, es decir, como una especie de compromiso entre libertad y necesidad. Precisamente por esta peculiar limitación de la causalidad universal en las ciencias que estudian comportamientos, es que ellas han tenido -y todavía incluso tienen - dificultades para ser admitidas en el campo de la ciencia en sentido estricto. Sin embargo, lejos de ser un obstáculo, la inclusión epistemológica del indeterminismo libre debería ser una motivación para la búsqueda de nuevas teorías.

Celina A. Lértora Mendoza clertora@cocinet.gov.ar 